\title{
Archives
}

\section{Le Service des archives de l'Université de Montréal et la formation des archivistes}

\section{MARCEL LAJEUNESSE}

Volume 46, numéro 2, 2017

$50^{\mathrm{e}}$ anniversaire de la DGDA

URI : https://id.erudit.org/iderudit/1040381ar

DOI : https://doi.org/10.7202/1040381ar

Aller au sommaire du numéro

Éditeur(s)

Association des archivistes du Québec (AAQ)

ISSN

0044-9423 (imprimé)

2369-9256 (numérique)

Découvrir la revue

Citer cet article

Lajeunesse, M. (2017). Le Service des archives de l'Université de Montréal et la formation des archivistes. Archives, 46(2), 81-90.

https://doi.org/10.7202/1040381ar
Résumé de l'article

Ce texte présente l'apport du Service des archives à l'Université de Montréal dans la seconde moitié du $\mathrm{XX}^{\mathrm{e}}$ siècle dans la mise en place de programmes universitaires en archivistique. Carol Couture, alors directeur du Service, a été l'instigateur du développement de l'enseignement de l'archivistique au sein de l'École de bibliothéconomie et des sciences l'information. Pour se faire, il a notamment mobilisé le Service des archives, fournissant ainsi le personnel enseignant ainsi qu'un milieu de stages, tous indispensables à la formation de nouveaux archivistes. 


\section{ANNIVERSAIRE DE LA DGDA}

\section{Le Service des archives de I'Université de Montréal et la formation des archivistes}

\section{MARCEL LAJEUNESSE}

Professeur associé, École de bibliothéconomie et des sciences de I'information, Université de Montréal

\section{INTRODUCTION}

La formation est un domaine qui a préoccupé la profession des archivistes au cours des dernières décennies. Elle a donné lieu au cours de la seconde moitié du $X X^{\mathrm{e}}$ siècle à des débats, à des confrontations, à la recherche de solutions. L'Unesco et le Conseil international des archives, créé en 1948, ont été les principaux instigateurs de la mise en place de programmes de formation des archivistes dans les décennies qui ont suivi.

\section{UN PEU D'HISTOIRE}

Le XIX siècle a vu un grand mouvement de développement de l'histoire et de ses méthodes. L'École des chartes, fondée à Paris en 1821, s'insère pleinement dans ce courant de redécouverte du passé. L'enseignement de l'École a été basé, dès ses débuts, sur les sciences auxiliaires de l'histoire (paléographie, sigillographie, diplomatique, etc.) si importantes pour 
I'étude du Moyen Âge occidental. II faut dire que les archives médiévales pèsent lourd en France avec leurs 200 kilomètres d'archives. L'École des chartes a fait école. Les instituts de Marburg en Allemagne et de Vienne en Autriche sont des créations issues de ce courant romantique de redécouverte de I'histoire en général et de I'histoire du Moyen Âge en particulier. II en fut de même en Italie et à Moscou en 1930. En réalité, il s'est établi un modèle de formation des archivistes dans l'Europe du XIXe siècle dans lequel l'érudition historique occupe toute la place et l'on peut dire que l'archiviste en un historien (Lajeunesse, 1986).

Ce modèle de formation était mal adapté aux pays neufs, dont font partie les États-Unis et le Canada. Le grand archiviste américain Theodore Schellenberg a affirmé que la formation donnée en Europe a peu d'applicabilité dans le travail de l'archiviste de son pays (Schellenberg, 1968). Quelle doit être cette formation et où doit-elle être dispensée? Au cours des décennies 1940, 1950 et 1960, le choix de l'école de bibliothéconomie ou du département d'histoire fut un thème récurrent de la littérature professionnelle (Evans, 1975). Un numéro de la revue American Archivist d'avril 1968 discutait des choix possibles. On y constatait d'abord que la profession d'archiviste avait échoué dans la mise en place d'un programme de formation adéquat, régulier et complet. Dispenser quelques crédits d'archivistique dans une université n'était pas une solution. Un des auteurs y affirmait que seule l'histoire peut contribuer à la formation d'un archiviste, bien qu'il constate, par ailleurs, l'indifférence des historiens et des départements d'histoire à la formation en archivistique. De son côté, Schellenberg voyait le problème différemment. Docteur en histoire, à l'emploi des Archives nationales depuis 1935, professeur d'archivistique à l'American University ainsi qu'aux écoles de bibliothéconomie notamment du Texas, Washington, Columbia, Illinois et Syracuse, il constatait que, bien que les principes et les techniques des deux professions, bibliothécaire et archiviste, soient distincts, ils sont, dans un sens, complémentaires. Et les objectifs des deux professions sont manifestement les mêmes, à savoir rendre disponibles les ressources de la recherche du pays.

Tout le débat tourne autour de la question suivante: I'archiviste est-il un historien et un érudit ou est-il un professionnel de l'information? Après avoir constaté ce qu'archivistes et bibliothécaires ont en commun, I'archiviste fédéral du Canada, W. I. Smith, se faisait en 1980 le promoteur d'une formation professionnelle commune pour les archivistes et les 
bibliothécaires dans les mêmes écoles universitaires, depuis qu'ils sont confrontés aux mêmes fonctions dans la gestion de l'information. Plusieurs sujets peuvent être partagés entre bibliothécaires et archivistes, notamment la nature et les principes de la gestion de l'information, le droit d'auteur, la reprographie, I'automatisation, la conservation, les services de référence, mais ces cours doivent être suivis d'une spécialisation en archivistique pour les futurs archivistes (Ross, 1980).

\section{L'ARCHIVISTIQUE À L'ÉCOLE DE BIBLIOTHÉCONOMIE AU COURS DES ANNÉES 1970}

À son programme de maîtrise, inauguré en 1970, I'École de bibliothéconomie ajouta en 1972 un cours au choix de 45 heures (3 crédits) intitulé Archives du Québec et du Canada. L'archiviste de I'Université, François Beaudin, entré en poste en avril 1972, assura ce cours pendant les années universitaires 1974-1975 et 1975-1976. II quitte l'Université le $1^{\text {er }}$ septembre 1976 et Carol Couture est nommé le 23 septembre directeur du Service des archives, selon la nouvelle appellation de la fonction. Le cours d'archivistique ne fut pas dispensé au cours de l'année universitaire 1976-1977.

Carol Couture reprit pendant I'année 1977-1978 l'enseignement de ce cours de trois crédits «portant sur la gestion des documents et le traitement des archives historiques» (Service des archives, 1977-1978). Le Service des archives reçut au cours de cette année-là 10 stagiaires de l'École de bibliothéconomie auxquels s'ajoutèrent six stagiaires du Collège de Maisonneuve et un stagiaire du Collège Lionel-Groulx provenant des programmes de bibliotechnique. La tâche du Service des archives s'alourdit l'année suivante. En effet, le Service a reçu en 1978-1979, en prolongement du cours dispensé par Carol Couture, 25 stagiaires de l'École à raison de 18 heures chacun à la division des archives historiques et un stagiaire en gestion des documents pour une durée de 15 jours. Ces stages donnaient lieu à la production d'inventaires. Citons pour exemples I'Inventaire du fonds Gaspard-Dauth (Isabelle Laperle-Dionne et Hélène Perron), I'Inventaire analytique du fonds Louvigny-de-Montigny (Marcel Boulet), le Répertoire numérique détaillé du fonds Édouard-Garand (Ginette Michaud) et celui du fonds de I'Institut agricole d'Oka (Marcel Boulet et Jacques Ducharme). 
Entretemps, I'École de bibliothéconomie était engagée depuis 1975 dans une profonde réforme de son programme de maîtrise. Dans le nouveau programme que l'Assemblée de l'École approuvait en 1977, de nouveaux buts et objectifs du programme étaient définis. On y retrouvait une explicitation du terme de spécialiste de l'information documentaire, qui désigne «les bibliothécaires, les documentalistes, les archivistes, les gestionnaires de documents, les analystes, les agents d'information, les cartothécaires, les audiovidéothécaires, etc., et les spécialistes de I'information scientifique et technique » (École de bibliothéconomie et des sciences de l'information, Guide de l'étudiant, 1980-1981).

Au cours de l'année 1979-1980, Carol Couture dispense le cours sur les archives du Québec et du Canada. Cette année-là, comme ce fut le cas au cours des années précédentes, les étudiants de la maîtrise de l'École de bibliothéconomie ont eu droit à une visite du Service des archives. En 1979-1980, le Service a supervisé le stage de deux étudiants de l'École, ainsi que huit étudiants du Collège de Maisonneuve et deux du Collège Lionel-Groulx.

\section{LE SERVICE DES ARCHIVES ET LA FORMATION DES ARCHIVISTES DANS LA DÉCENNIE 1980}

Le nouveau programme de maîtrise, approuvé par les professeurs de l'École en 1977, fut entériné par les différentes instances de l'Université en 1979 et dispensé pour la première fois au cours de l'année universitaire 1980-1981. Ce nouveau programme offrait en seconde année de maîtrise neuf concentrations en divers domaines de la bibliothéconomie et des sciences de l'information; ceux-ci comprenaient un cours théorique, un cours d'étude de cas et un séminaire de recherche auquel se greffait un stage obligatoire de 20 jours ou 140 heures. L'archivistique ne faisait pas l'objet d'une concentration, mais passait d'un à deux cours dans la liste des cours interdisciplinaires, l'un intitulé Archivistique (traitement des archives historiques) et l'autre, Gestion de la documentation (traitement des documents administratifs). Ces deux cours étaient dispensés par le directeur du Service, Carol Couture.

De 1980 à 1982, les archivistes du Service des archives, Carol Couture au premier chef, étaient accaparés par la rédaction du livre Les archives au $X X^{e}$ siècle: une réponse aux besoins de l'administration et de la recherche 
qui parut en 1982. Cela n'empêche pas Carol Couture de passer au tournant des années 1980 en vitesse supérieure concernant la formation en archivistique. En décembre 1979, il présente au directeur Daniel Reicher un projet d' " orientation en archivistique » qui pouvait regrouper 13 crédits parmi les cours de la section des cours interdisciplinaires. Aux deux cours d'archivistique du nouveau programme, on pouvait y ajouter un stage, prévu lui aussi dans le programme. II suggérait, de plus, I'ajout de cours aux HEC en administration et de cours en sciences politiques concernant les institutions politiques canadiennes et québécoises. Couture revient à la charge à ce sujet en juin 1981. Un sous-comité du Comité des études de l'École étudie les demandes de Couture et il propose l'ajout d'une concentration archivistique comprenant les deux cours déjà au programme, un stage et l'ajout d'un troisième cours portant sur la recherche en archivistique. Cette nouvelle concentration était adoptée en 1982.

Les premières années de la décennie 1980 ont été une période propice à la mise en place de programmes de formation en archivistique dans les universités québécoises. L'adoption de deux lois, la Loi sur l'accès aux documents des organismes publics et la protection des renseignements personnels (RLRQ, chapitre A-2.1) et la Loi sur les archives (RLRQ, chapitre A-21.1) suscite le besoin de professionnels capables de doter les organismes d'une classification et d'un calendrier de conservation nécessaires à une saine gestion de leurs documents (Arès, Couture et Gagnon-Arguin, 1987).

Carol Couture organise à l'École de bibliothéconomie à I'hiver 1982 une journée d'étude qui a été un moment important pour la promotion de l'archivistique à l'École. Cette journée d'étude rassemble professeurs, étudiants, chercheurs et archivistes pour faire le point sur l'archivistique, son développement au Québec et au Canada et la situation de l'enseignement de ce domaine au Canada et dans le monde.

En 1982-1983, I'Association des archivistes du Québec offrait à ses membres, en collaboration avec l'École de bibliothéconomie, les deux cours que Carol Couture dispensait déjà dans le programme de l'École. Cela démontrait d'une manière manifeste le besoin de formation ressenti dans la profession.

Parallèlement à la concentration en archivistique en maîtrise, en tant que directeur intérimaire de l'École, je mis sur pied au début de l'automne 1982 un comité chargé d'étudier l'opportunité de créer un certificat 
de premier cycle en archivistique, composé de dix cours de trois crédits chacun. L'Assemblée de département approuvait à l'unanimité le 19 novembre 1982 le rapport du comité et recommandait la création du certificat en suggérant le démarrage de celui-ci à l'automne 1983. Ce certificat avait pour but de donner une formation professionnelle de base en archivistique en rendant les étudiants aptes à appliquer les principes et les méthodes propres à l'archivistique ou de fournir, en plus, la possibilité d'ajouter ce certificat comme mineur d'un baccalauréat majeur-mineur. Le certificat en archivistique eut, dès son démarrage en septembre 1983, un grand succès, avec une inscription de 104 étudiants.

Il est intéressant de relire dans les rapports annuels du directeur du Service des archives les portions relatives à la formation des archivistes. Chacun des rapports de Carol Couture y fait référence. Mais c'est dans les rapports de 1982-1983 et celui de 1983-1984 que la contribution du Service est la plus développée. Dans le rapport de 1982-1983, on y lit que «le Service des archives s'est de plus en plus impliqué dans un domaine qui l'a toujours intéressé au plus haut point, soit la formation » (Service des archives, 1982-1983). Cet intérêt a pris plusieurs formes. Le directeur du Service a dispensé de nombreux cours d'archivistique tant à I'École de bibliothéconomie qu'à l'Association des archivistes du Québec. Il a participé à des comités de l'École qui ont recommandé la création d'une concentration en archivistique en maîtrise et d'un programme de certificat en archivistique au premier cycle. Le Service a reçu de nombreux stagiaires provenant de l'École de bibliothéconomie. II a pu compter sur la collaboration de ses collègues archivistes du Service pour dispenser des cours d'archivistique à l'École, pour encadrer les stagiaires ou pour siéger sur des comités de l'École portant sur la gestion des programmes d'archivistique. II écrit:

Quand on prend tout cela en considération, on constate que le Service des archives s'implique sans cesse davantage dans la formation en archivistique espérant ainsi faire avancer ce dossier important. À compter de septembre prochain, beaucoup de moyens seront en place; il restera à les mettre en œuvre et à voir si les besoins de formation exprimés étaient véritables. (Service des archives, 1982-1983)

En 1983-1984, le directeur du Service réaffirmait «que la formation en archivistique a été un élément majeur parmi les activités du Service des 
archives » pendant l'année qui venait de se terminer (Service des archives, 1983-1984). II constatait que c'est grâce à l'expertise du Service que celui-ci peut offrir et à un intérêt marqué pour la formation que le Service a pu être associé d'aussi près à ce projet qui relève plus de l'enseignement que de l'administration. II mentionnait que quatre personnes du Service donnaient des cours à l'École de bibliothéconomie, que le personnel était affecté à l'encadrement de stagiaires, que le Service servait de milieu d'étude et de laboratoire pour les étudiants en archivistique à l'École de bibliothéconomie. II concluait en affirmant «le Service est très présent sur cette scène et compte bien y demeurer » (Service des archives, 19831984). Ainsi, selon lui, un service d'archives universitaire ne peut pas être mieux intégré à son environnement.

Étant donné l'ampleur que prend le secteur de l'archivistique et qu'il n'y a aucun professeur régulier en archivistique à l'École de bibliothéconomie, la Faculté des arts et des sciences, autorise, en juin 1984, à la demande de l'École, la création d'un poste de professeur mi-temps. Après étude du dossier, les professeurs de l'École proposent la nomination de Carol Couture comme professeur agrégé mi-temps à partir du premier janvier 1985, comme étant le meilleur candidat en raison de sa compétence, de son esprit de collaboration avec l'École, son dynamisme et sa grande disponibilité (École de bibliothéconomie et des sciences de l'information, Procès-verbaux, 21 juin 1984). De 1985 à 1988, Carol Couture, professeur à mi-temps, demeure directeur à mi-temps du Service des archives de l'Université.

En février 1985, Carol Couture regrette que la concentration en archivistique en seconde année de maîtrise ne soit pas appuyée, comme c'est le cas pour les autres concentrations, par un cours obligatoire en archivistique en première année de maîtrise. Le comité des études de l'École propose alors une modification du programme par l'ajout d'un nouveau cours de deux crédits intitulé Introduction à l'archivistique, en première année. Ce nouveau cours obligatoire fut mis au programme en 1986.

Compte tenu du succès des programmes d'archivistique, deux postes de chargé de formation pratique, I'un à plein temps et l'autre à mi-temps, sont ajoutés en 1985 au personnel de l'École de bibliothéconomie et des sciences de l'information (EBSI), nouvelle appellation de l'École depuis 1984. Un deuxième poste de professeur en archivistique s'ajoute en 1987: Louise Gagnon-Arguin, chargée de formation pratique, devient 
chargée d'enseignement et elle deviendra professeure adjointe en 1990 lors de l'obtention de son doctorat. L'autre poste de chargé de formation pratique est transformé en un poste de coordonnateur des stages en archivistique: Florence Arès est chargée de cette responsabilité.

Le rapport annuel du Service des archives est signé pour l'année 19841985 par Jean-Yves Rousseau, nouveau directeur adjoint du Service. On y fait mention que six étudiants de maîtrise et quatre étudiants du certificat ont été stagiaires au Service au cours de cette année-là. Carol Couture signe les rapports de 1985-1986 et 1986-1987. En 1985-1986, cinq étudiants de la maîtrise et six du certificat firent leur stage au Service, tandis que l'année suivante, il y eut deux étudiants de la maîtrise et sept du certificat.

Le premier juin 1988, Carol Couture devient professeur agrégé plein temps à l'École de bibliothéconomie et des sciences de l'information et il quitte alors le Service des archives. À partir du départ de Carol Couture en 1988, on constate que le domaine de la formation des archivistes devient secondaire dans les rapports annuels du Service et il disparaît en quelque sorte des intérêts du Service au tournant des années 1990.

En devenant professeur de carrière, Carol Couture devait développer un programme de recherche, la recherche étant une fonction inhérente à sa nouvelle fonction. Au cours de la décennie 1980, la recherche en archivistique, distincte de la recherche en histoire, était peu pratiquée dans notre milieu. Le contexte des sciences de l'information de l'École de bibliothéconomie et des sciences de l'information s'avérait un terreau propice au développement de la recherche en archivistique. La première étape de la constitution d'un programme de recherche fut la création, en 1987, du Groupe interdisciplinaire de recherche en archivistique (GIRA) par Carol Couture, Jacques Ducharme et Jean-Yves Rousseau. Le GIRA visait, entre autres objectifs, à participer à l'établissement d'un programme de recherche et à apporter une contribution théorique à la discipline et à la profession. En 1990, eut lieu le premier colloque du GIRA sous le titre de La place de l'archivistique dans la gestion de I'information: perspectives de recherche. Le GIRA en est, avec le colloque de 2014, à son $7^{\mathrm{e}}$ colloque quadriennal. En 1989, Carol Couture initie un vaste programme de recherche en trois volets: législation archivistique et politiques nationales d'archives; principes et fonctions archivistiques; enseignement et recherche en archivistique, qui s'est étendu pendant toute la décennie 1990 et qui a donné lieu à de nombreuses publications tant au plan national qu'au plan international. 


\section{CONCLUSION}

Il y avait un cours d'archivistique dans le programme de maîtrise de I'École de bibliothéconomie et des sciences de l'information dès le début de la décennie 1970, mais celui-ci était considéré, en tant que cours au choix, comme un ajout de culture professionnelle dans la formation des bibliothécaires. Quand on scrute les archives de l'École et celles du Service des archives, on constate que c'est vraiment au cours de la décennie 1980, principalement entre 1979 et 1988, que s'est développé l'enseignement de I'archivistique par l'introduction de programmes d'archivistique cohérents tant au second qu'au premier cycle, par l'engagement de professeurs-chercheurs.

Ce développement est sans conteste l'œuvre du directeur du Service des archives, Carol Couture, qui n'a cessé de revenir à la charge, de bombarder le directeur de l'École de demandes, de convaincre les professeurs de l'École de l'intérêt de mettre en place des programmes d'archivistique, d'inscrire ou de faire inscrire des points à l'ordre du jour de l'assemblée de département. II est évident qu'il avait donné au Service des archives, dès le tournant des années 1980, une mission concernant l'implantation de la formation des archivistes à l'Université de Montréal et il s'est rapidement convaincu que l'École de bibliothéconomie et des sciences de l'information constituait le terreau idéal pour y créer et développer des programmes en ce domaine. II faut dire que la période s'y prêtait à cause de la législation en archivistique. C'est aussi en ces années qu'on a observé à l'École l'essor des sciences de l'information qui permettait d'inclure des domaines connexes à la bibliothéconomie.

Carol Couture a mobilisé dans cette mission de formation des archivistes, au cours de ces années, le personnel du Service des archives pour donner des cours, pour prononcer des conférences dans les cours, pour superviser des stages. II avait tout d'abord convaincu ses supérieurs hiérarchiques successifs, les Secrétaires généraux de l'Université, Jacques Boucher d'abord, puis Michel Lespérance, de l'importance de cette action. Ceux-ci lui ont toujours accordé un appui solide. Les rapports annuels du Service permettent de suivre la pensée et l'action de Couture dans ses rapports avec l'École et de suivre la progression de son projet. II est indéniable que Carol Couture a démontré tout au long de ces années une constance et une détermination dans la poursuite de son projet de former des archivistes professionnels. Pendant la décennie 1980, le Service des archives fut mobilisé dans la poursuite de cet objectif. 


\section{BIBLIOGRAPHIE}

ARÈS, F., COUTURE, C. et GAGNON-ARGUIN, L. (1987). L'archivistique à l'École de bibliothéconomie et des sciences de l'information. Argus 16(1), 9-11.

ÉCOLE DE BIBLIOTHÉCONOMIE ET DES SCIENCES DE L'INFORMATION. (1970-1986). Procès-verbaux de l'assemblée de département. Fonds de l'École de bibliothéconomie et des sciences de l'information (E0006). Université de Montréal, Division de la gestion de documents et des archives.

ÉCOLE DE BIBLIOTHÉCONOMIE ET DES SCIENCES DE L'INFORMATION. (1977/1978-1986). Guide de l'étudiant. Fonds de l'École de bibliothéconomie et des sciences de l'information (E0006). Université de Montréal, Division de la gestion de documents et des archives.

EVANS, F. B. (1975). Archival training in the United States: An unresolved problem. Archives et bibliothèques de Belgique 46(36), 518-547.

LAJEUNESSE, M. (1986). L'archivistique: une science de l'information à la recherche de son milieu de formation. Archives 18(3), 35-47.

LAJEUNESSE, M. (2002). L'École de bibliothéconomie et des sciences de I'information (EBSI) de I'Université de Montréal 1961-2003: quatre décennies de formation des spécialistes de l'information au Québec et au Canada français. Documentation et bibliothèques 48(4), 125-135.

ROSS, I. (1980). Library-Archive Relations: The question of education. Canadian Library Journal 37(1), 43.

SCHELLENBERG, T.R., (1968). Archival training in library schools. American Archivist 31(2), 155.

SERVICE DES ARCHIVES. (1971/1972-1989/1990). Rapports annuels du Service des archives. Fonds de la Division de la gestion de documents et des archives (D0006). Université de Montréal, Division de la gestion de documents et des archives. 